\title{
EXPLOSIVE COSMOGONY AND THE QUASI-STEADY STATE COSMOLOGY
}

\author{
G. BURBIDGE \\ University of California, San Diego \\ Center for Astrophysics 83 Space Sciences 8 Dept. of Physics \\ La Jolla, CA 92093-0424
}

\section{The Historical Setting}

Modern cosmology began with the realization that there were solutions to Einstein's theory of gravity discovered by Friedmann and Lemaitre which when combined with the redshift distance relation of Hubble and others could be interpreted as showing that we live in an expanding universe. By 1930, the scientific establishment and many of the lay public believed this. It was then only elementary logic to argue that if time reversal was applied, the universe must originally have been so compact that we could talk of a beginning. Lemaitre tried to describe this state as the "Primeval Atom." For a decade or so after the war, Gamow, Alpher and Herman and other leading physicists explored this dense configuration trying to make the chemical elements from protons and neutrons. They soon learned that this was not possible because of the absence of stable masses of five and eight, but they also realized that if such an early stage had occurred the universe would contain an expanding cloud of radiation which would preserve its black body form. Dicke and his colleagues in Princeton rediscovered this idea and decided to try and detect the radiation. Penzias and Wilson found such a radiation field, and COBE has demonstrated that it has a perfect black body form out to radio wavelengths. This history of the discovery together with the fact that the light elements $\mathrm{D}, \mathrm{He}^{3}$ and $\mathrm{He}^{4}$ in about the right amounts can be made in a hot big bang has led to the widely held, but simplistic view, that the standard cosmology - the hot big bang - is correct.

As the belief in this theory has grown, with some of its popularity coming from the fact that a beginning is a main theme of western religion, it has become progressively harder to argue against it.

\section{Real Objections to the Big Bang Model}

\subsection{THE EARLY DISCOVERY AND THE HE/H PROBLEM}

What was not properly understood by those who first discussed the early universe was that McKellar had already discovered the microwave radiation in 1941 (he obtained a temperature of $2.3^{\circ} \mathrm{K}$ setting as a lower limit $1.8^{\circ}$, and as an upper limit $3.4^{\circ} \mathrm{K}$ for the interstellar temperature) (McKellar 1941). It is also clear that the only reason why the physicists decided to invoke a dense configuration in an early universe was to find a place with a plentiful source of neutrons. Since the model failed to 
explain the building of nearly all of the chemical elements (which we now know following Hoyle were made in the stars) the model might well have been dropped. This is especially clear when it is also pointed out that in the 1950s both Bondi, Gold and Hoyle (1955) and independently, Burbidge (1958) pointed out that if the observed abundance of He was obtained by hydrogen burning in stars, there must have been a phase in the history of the universe when the radiation density was much higher than the energy density of starlight today. The very striking fact is that if we suppose that if $\rho$ is the density of visible matter in the universe, with a value of about $3 \times 10^{-31} \mathrm{gm}^{-3}$, and that the $\mathrm{He} / \mathrm{H}$ ratio by mass in it is 0.244 , then the energy which must have been released in producing $\mathrm{He}$ is $4.39 \times 10^{-13} \mathrm{erg} \mathrm{cm}^{-3}$. If this energy is thermalized, the black body temperature iturns out to be $T=2.76^{\circ} \mathrm{K}$. This value is astonishingly close to the value of $2.73^{\circ} \mathrm{K}$ observed by COBE.

This simple agreement of two measured quantities makes no allowance for the expansion of the universe that must necessarily have taken place during the production of helium, which would act to reduce the temperature. However, it does show that unless it is dismissed as a coincidence which all big bang believers must do, there is likely to be an explanation of the microwave radiation in terms of straight forward astrophysics involving hydrogen burning in stars.

It can be argued therefore that this line of reasoning completely refutes the popular view that the discovery of the microwave radiation is proof that a big bang occurred.

The usual rebuttal to this argument is that it is the blackbody nature of the radiation that is important, not the value of the temperature, and that in any alternative scheme it is the thermalization process of the radiation that is the weak link.

The counter to this argument is that in the standard model generation of the black body radiation is traced to the decay of the false vacuum energy in the inflationary scenario. But as we shall show this whole approach is a gross extrapolation beyond known physics.

\subsection{THE ARBITRARY NATURE OF THE PHYSICS IN THE EARLY UNIVERSE}

This can be seen by reversing the time axis associated with the expansion. As the universe shrinks the radiation energy begins to dominate the matter and ultimately the matter is broken down into quarks. We now move out of the realm of known physics. A further contraction by a factor of about $10^{10}$ is invoked leading to what is called a "phase transition" in which everything is converted into a new kind of so-called scalar particle. These scalar particles are supposed to interact together to produce what is described as a "false vacuum" maintaining positive energy at all costs. This false vacuum consumes space-time in a process of deflation - this is the inflation epoch of Guth and Linde when time is reversed. The consuming of spacetime leads to what? To a quantum transition to somewhere else!

If these words are not understandable to the layman it is because they are not understandable to physicists either, however "nice" the idea of inflation may be. And if not inflation what else? The problem is the resistance to the idea (of Dirac) of particles of negative energy. While the energetics are still outside the realm of known physics, the existence of a negative energy field will permit entirely new positives to form with the new positives compensating those of negative energy with what we can refer to as creation events in which energy is conserved. This approach is already preferable.

The great importance of it is that there is much observational evidence pointing to such creation events. This evidence is the cosmogonical basis for the cosmological quasi-steady state theory which Dr. Narlikar will outline. 
In the classical big bang cosmology all of the discrete objects are supposed to have arisen from density fluctuations in the early universe. While there is no direct evidence at all for this, evidence of the other kind is widespread. None of this evidence is understood within the framework of the big bang cosmology.

\section{The New Observational Evidence}

Starting about forty years ago, Ambartsumian $(1958,1965)$ proposed that groups and clusters of galaxies that appear to be expanding are doing just that. They are systems of positive total energy. This idea was resisted by Oort and others on the ground that the galaxies must be very old, but if they were being ejected in expanding associations this could not be correct. To bind the groups and clusters dark matter was invoked. This was one of the early arguments for the presence of dark matter.

For clusters which are really relaxed and obviously stable this is a perfectly good argument, but for many, if not the majority of clusters which show every sign of instability it seems likely that the virial does not hold and they are coming apart. There is only limited evidence, of course, that galaxies in general are $\sim 10^{10}$ years old. We now accept the fact that many galaxies have very young components, and perhaps whole galaxies may be young.

Also in the $1960 \mathrm{~s}$, it became clear that the nuclei of galaxies often give rise to explosive outbursts (Burbidge, Burbidge \& Sandage 1963). The most powerful of these are the radio outbursts which can generate $10^{60}-10^{62}$ ergs largely in the form of relativisitic particles. With the discovery of the quasi-stellar objects came the evidence that many of the radio emitting QSOs lie so close to comparatively nearby galaxies that the configurations cannot be accidental, although the QSOs have much larger redshifts then the galaxies. Thus it follows that the QSOs must have been ejected from the galaxies and must have large intrinsic redshift components. Evidence of this kind has been obtained in profusion over the last twenty-five years by Arp, ourselves and others (cf Burbidge, Burbidge \& Sandage 1963, Arp 1987, Burbidge 1996).

Most recently clear cut evidence showing that X-ray emitting QSOs are being ejected from nearby active galaxies (NGC 4258, 1068, 3516, 5548, etc.) has been obtained. Arp will describe some of the new results.

How have these observations been explained in the classical picture. Two lines of attack hae been developed. The paradigm put forward to explain the activity in galactic nuclei is that the energy is gravitational in origin and is generated from matter falling into the center which contains a massive black hole and an accretion disk (cf Rees 1984). This paradigm is never tested but continuously asserted. In our early calculations (Hoyle et al. 1964) we showed how implausible this was. As far as the evidence for QSOs etc. ejected from galaxies is concerned the position that is taken is that the data are all suspect and therefore need not be explained.

On the other hand, we accept the observational evidence of expanding groups and clusters, and ejection phenomena involving high energy events from the centers of radio galaxies and many other active galaxies which shows that dense objects are ejected from the nuclei of already existing galaxies.

Thus we believe that galaxies beget galaxies, not that they are made from initial density fluctuations in a big bang universe.

The cosmological model which is based on this cosmogony is the quasi-steady state cosmology. This is the model developed by Hoyle, Burbidge and Narlikar which has been published in a series of papers recently (Hoyle, Burbidge and Narlikar 1993, 1994ab, 1995): 
In this theory there can be particles with negative energy. The particles exert a negative pressure. This negative pressure produces the expansion of the universe, not an initially assumed explosion. The universe goes through repeated cycles of expansion and contraction, each expansion driven by negative pressure inside many localized regions (galaxies) where a large amount of creation occurs.

These cycles with a total period of about $40 \times 10^{9}$ years are superposed on an overall slow expansion with a time scale $\sim 10^{12}$ years.

Dr. Narlikar will discuss the ways in which we can understand the abundances of the light elements and the microwave background radiation within the framework of this theory.

\section{References}

\section{References}

Ambartsumian, V. A. 1958, Solvay Conf. Report,(ed R. Stoops) Bruxelles.

Ambartsumian, V.A. 1965, "Structure and Evolution of Galaxies" Proc. 13th Solvay Conf. on Physics, University of Brussels (Wiley Interscience)

Arp, H.C. 1987, “Quasars, Redshifts \& Controversies" (Interstellar Media, Berkeley).

Bondi, H., Gold, T. \& Hoyle, F. 1955, Observatory Mag., 75, 80.

Burbidge, G. 1958, P.A.S.P., 70, 83.

Burbidge, G. 1996, A\&A, 309,9.

Burbidge, G., Burbidge, M. \& Sandage, A.R. 1963, Rev. Mod. Phys. 35, 947.

Hoyle, F., Burbidge, G. \& Narlikar, J.V. 1993, ApJ, 410, 437.

Hoyle, F., Burbidge, G. \& Narlikar, J.V. 1994a, MNRAS, 267, 1007.

Hoyle, F., Burbidge, G. \& Narlikar, J.V. 1994b, A\&A, 289, 729.

Hoyle, F., Burbidge, G. \& Narlikar, J.V. 1995, Proc. Roy. Soc., A, 448, 191.

Hoyle, F., Fowler, W.A., Burbidge, G., \& Burbidge, M. 1964, ApJ, 139, 909.

McKellar, A. 1941, Pub. Dom. Astrophy. Observatory, Vol. 7, No. 15

Rees, M.J. 1984, ARA\&A, 22, 471. 